\title{
Éditorial:
}

\section{Les personnes âgées atteintes de troubles cognitifs et vivant seules sont-elles en sécurité?}

Avec le vieillissement de la population, deux tendances convergentes sont de plus en plus manifestes dans les nations industrialisées: l'augmentation du nombre de personnes âgées vivant seules, et l'augmentation du nombre de personnes atteintes de la maladie d'Alzheimer ou d'autre formes de démence (Weber, Fox, \& Burnette, 1994; Chappell, 1991). L'Étude sur la santé et le vieillissement au Canada (ESVC) (Étude sur la santé et le vieillissement au Canada, 1994) rapporte que 29 pour cent des Canadiens atteints de démence et qui ne sont pas institutionnalisés vivent seuls. Les personnes atteintes de troubles cognitifs ou de démence et qui vivent seules sont plus susceptibles d'être placées dans des établissements de soins que celles qui résident avec une autre personne. Les décisions d'institutionnalisation prises par les aidants naturels sont souvent fondées sur les difficultés qu'ils éprouvent à assurer les soins requis et sur leur propre évaluation du besoin de la personne âgée pour des soins plus spécialisés. En fait, les caractéristiques des aidants constituent un meilleur prédicteur des décisions d'institutionnalisation que les caractéristiques de la personne aidée (Colerick \& George, 1988; Gold, Reis, Markiewicz, \& Andres, 1995). La situation est aggravée par le fait que les organismes de soutien à domicile ne sont pas en mesure de déterminer qui sont les personnes les plus à risque et donc de faire porter leurs efforts sur ces personnes. Il en découle que des personnes âgées atteintes de troubles cognitifs, mais qui désirent rester dans leur milieu, peuvent être institutionnalisées sans raison ou de façon prématurée du fait des difficultés anticipées par des parents inquiets et parce qu'elles reçoivent une assistance insuffisante des organismes de soutien à domicile.

L'ESVC (Ostbye \& Crosse, 1994) estime à plus de 3,9 milliards de dollars par année le coût net de la démence au Canada. Le coût annuel par patient est de 8096 (total des services rémunérés et non rémunérés) pour ceux qui vivent à domicile, alors qu'il s'élève à $28100 \$$ par personne en institution. De toute évidence, il est plus économique de maintenir les personnes dans leur milieu. Par contre, le fait de laisser des personnes atteintes de troubles cognitifs dans leur milieu comporte également des risques (et des conséquences économiques). Ces risques comprennent le danger de dommages ou même de décès résultant d'erreurs de médication, de malnutrition, de déshydratation, d'incendies, ou d'autres conséquences tout aussi graves.

La principales inquiétude des professionnels de la santé à propos de leurs patients atteints de troubles cognitifs et vivant seuls touche à leur sécurité. 
Plusieurs professionnels ressentent un conflit entre leur désir d'assurer un environnement sécuritaire à leurs patients (par ex., une institution) et leur désir de respecter le droit de leurs patients de vivre où ils le veulent. Considérant l'importance et l'ampleur de ce problème, on s'attendrait à retrouver de la documentation considérable décrivant la nature et la fréquence des difficultés rencontrées par ces personnes âgées dans leur milieu. Pourtant, les seules recherches effectuées dans ce contexte portent sur la conduite automobile et le risque d'accident de la route. Ces études ne sont pas concluantes; certaines montrent une augmentation du risque d'accident pour les personnes atteintes de la maladie d'Alzheimer (Trobe, Waller, Cook-Flannagan, Teshima, \& Bieliauskas, 1996); d'autres, non (Drachman, Swearer, \& groupe d'étude collaborative, 1993). Cependant, les accidents d'automobile sont des événements relativement rares et aucune recherche n'a porté sur le lien entre les autres conséquences néfastes et les troubles cognitifs affectant les personnes âgées vivant dans leur milieu. Par contre, les conséquences de l'institutionnalisation ont fait l'objet de vastes recherches.

Dans les études visant à prédire l'institutionnalisation, les modèles prédictifs sont fondés sur le bien-être des soignants ou la charge qui leur incombe, ou sur l'état physique ou cognitif du patient. Colerick et George (1988), par exemple, signalent que le fait pour une personne d'être mariée constitue l'un des principaux prédicteurs permettant de déterminer si une personne atteinte de la maladie d'Alzheimer sera institutionnalisée un an plus tard. Les sujets dont le soignant était une personne autre que le conjoint et soumise à un stress supplémentaire (dans la plupart des cas, une fille occupant un emploi) étaient plus susceptibles d'être institutionnalisés, sans égard à leur condition physique. Ces découvertes importantes ont été confirmées plus tard par plusieurs autres études. Il est intéressant de constater que le recours aux services communautaires n'a pas été reconnu comme un facteur majeur dans la prédiction de l'institutionnalisation (Cohen et al.,1993; McFall \& Miller, 1992; Newman, Struyk, Wright, \& Rice, 1990). Une seule étude (Webber et al., 1994) a comparé le taux d'utilisation des services communautaires des personnes atteintes de la maladie d'Alzheimer et vivant seules à celui des personnes qui habitaient avec une autre personne. L'étude a montré que les personnes atteintes d'Alzheimer font rarement appel aux ressources communautaires et que cette assistance est plus susceptible d'être requise par les aidants. De plus, le type de services requis était différent pour les deux groupes; les personnes atteintes d'Alzheimer et vivant seules faisaient plus souvent appel aux services de repas, de travaux ménagers, de gestion de cas et de soignants non familiaux, tandis que les personnes qui vivaient avec d'autres consultaient davantage les médecins et faisaient davantage appel aux centres de jour. Il semble donc que les services communautaires jouent un rôle différent pour les aidants et pour les bénéficiaires âgés. Lorsque l'on considère les caractéristiques de l'aidant principal, la différence entre les deux groupes réside surtout dans le fait que les personnes vivant seules sont beaucoup moins susceptibles que 
les autres d'avoir leur conjoint comme aidant principal. Il est intéressant de noter que les personnes vivant seules sont aussi moins susceptibles d'avoir une fille comme personne aidante. Si l'on considère ces constatations conjointement avec celles de Colerick et Greorge (1986), le profil suivant semble se dessiner: les personnes âgées atteintes de troubles cognitifs qui ont leur conjoint comme aidant présente la plus forte probabilité de demeurer à domicile. Après le décès de leur conjoint, cependant, ceux qui vivent seuls et dont les aidants sont des membres de la famille sont plus susceptibles d'être institutionnalisés que ceux dont les aidants ne font pas partie de la famille. Les inquiétudes ressenties pour la sécurité de la personne âgée par les membres de la famille qui agissent comme aidants et leurs propres facteurs de stress sont les principaux facteurs menant à l'institutionnalisation.

Qu'est-ce que la recherche et la législation relatives à l'incapacité mentale et à l'inaptitude nous apprennent quant à la prédiction de la sécurité des personnes atteintes de troubles cognitifs? De façon générale, une personne est considérée apte ou habilitée à prendre une décision sur un sujet particulier lorsqu'elle est en mesure de comprendre le sujet sur lequel une décision est requise, et qu'elle est capable de saisir les conséquences de sa décision. Il existe une présomption d'aptitude jusqu'à ce que l'inaptitude ait été prouvée de manière probante. La personne est alors déclarée inapte, de façon à ce qu'on puisse désigner un tuteur ${ }^{1}$ et ainsi fournir l'assistance requise pour assurer que les besoins financiers et personnels de la personne sont satisfaits. Bien que la législation varie d'une province à l'autre, l'approche générale est la suivante: l'intervenant qui allègue l'inaptitude doit demander au Tribunal de déclarer la personne inapte et de désigner le requérant à titre de tuteur. Habituellement, le tribunal demandera l'opinion d'un ou plusieurs professionnels de la santé quant à l'inaptitude. La plupart des lois sur l'inaptitude établissent une différence entre l'aptitude à gérer ses affaires financières et l'aptitude à prendre des décisions d'ordre personnel. En Ontario, la Loi sur la prise de décision au nom d'autrui précise les procédures et les critères qui doivent être appliqués dans l'évaluation et l'établissement de l'inaptitude légale. Un évaluateur désigné par le tribunal doit établir l'inaptitude à partir d'observations directes et de renseignements fournis par un tiers informateur quant à la capacité de la personne à prendre soin d'elle-même. Cette approche est utilisée en lieu d'une évaluation formelle parce qu'il n'existe aucune échelle permettant de prédire si une personne est susceptible d'encourir des dommages au plan personnel ou financier en raison de troubles cognitifs. La validité des échelles d'aptitude mentale a été établie en comparant la performance de personnes ayant besoin d'un degré de supervision différent sur ces échelles (D'Andrea, Goldberg, Searight, Gilner, \& Katz, 1991) et en établissant une corrélation entre ces performances, les échelles de comportement complétées par les soignants et les décisions d'équipes multidisciplinaires (Rutman \& Silberfeld, 1992). Ces approches de validation sont limitées par le fait qu'aucune d'elles n'incorpore des indices d'aptitude que l'on pourrait 
qualifier de "mesures-étalon". L'occurrence réelle d'actes résultant en des blessures ou des pertes de biens n'a été utilisée pour valider jamais ces échelles. Le développement d'une échelle permettant de mesurer l'aptitude d'une personne sur la base de résultats en terme de dommage ou de sécurité représenterait un apport important, tant clinique que théorique, au domaine de l'étude de l'aptitude mentale.

Quel est l'élément le plus important à connaître des personnes âgées atteintes de troubles cognitifs et de leur environnement pour permettre de prédire si elles peuvent encourir des dommages? À partir d'études déjà effectuées, les facteurs suivants pourraient constituer les prédicteurs de dommages les plus importants pour ces personnes: (1) l'étiologie de leur trouble cognitif; (2) le type de déficits cognitifs dont ils sont atteints; (3) les troubles de comportements qu'ils manifestent; et (4) les ressources sociales existantes. Nous allons passer brièvement en revue les justifications qui nous permettent de définir ces quatre facteurs comme primordiaux dans la prédiction des dommages.

En ce qui concerne l'étiologie des troubles cognitifs, un diagnostic de Maladie d'Alzheimer (MA), par exemple, peut être considéré comme un facteur de risque important puisqu'il s'agit d'une affection progressive qui se traduit par une diminution constante de la conscience de soi et de la capacité d'apprentissage. Des études antérieures (Tierney, Szalai, Snow et al., 1996; Tierney, Szalai, Snow, \& Fisher, 1996; Jacobs et al., 1995) ont montré que nous pouvions prédire le développement et la progression de la MA; toutefois, il n'existe aucune étude ayant pour but de démontrer que les patients atteints de MA sont plus vulnérables aux dommages à différents stades d'évolution de la maladie que des patients atteints d'autres formes de troubles cognitifs. Conclure que les personnes atteintes de la MA subiront plus de dommages que celles atteintes d'autres formes de troubles cognitifs nous semble toutefois relever de la présomption, pour les raisons qui suivent. En premier lieu, l'évolution de la MA est variable, et des personnes à des stades différents de la maladie peuvent présenter des écarts dans leur capacité à éviter les dommages. En second lieu, les personnes atteintes de la MA peuvent être moins portées à s'engager dans des activités à risque que des personnes atteintes d'autres formes de troubles cognitifs et dont les facultés cognitives sont moins affectées. Par exemple, une personne atteinte de la MA pourrait ne jamais décider d'utiliser l'escalier du sous-sol, parce qu'elle ne se souvient pas de ce qui s'y trouve. Par contre, la personne qui se souvient de ce qui se trouve au sous-sol pourrait décider impulsivement d'aller chercher cette chose sans prendre les mesures appropriées pour éviter une blessure, c'est-à-dire, allumer la lumière, tenir la rampe, descendre une marche à la fois, enlever les obstacles, etc.

Parce que nous ne savons pas si un diagnostic particulier de démence signale l'émergence d'un risque, ou lequel du stade de la maladie ou de l'existence de déficits cognitifs spécifiques permet le mieux de prédire les dommages, nous croyons que les fonctions cognitives constituent le second prédicteur le plus important. Les dommages subis par une personne peu- 
vent être dus à la présence de déficits cognitifs spécifiques ou à une combinaison spécifique de déficits. Les capacités cognitives jugées importantes au fonctionnement sécuritaire dans le milieu comprennent la mémoire, le langage, l'attention et la concentration, le raisonnement, la solution de problèmes, la planification, la mise en séquence et les fonctions exécutives.

Les comportements des aînés peuvent aussi constituer les facteurs importants dans la prédiction des dommages. Les caractéristiques du comportement et des habiletés fonctionnelles considérées comme des prédicteurs importants des dommages sont les comportements psychotiques (incluant les illusions et les hallucinations), les difficultés à accomplir les activités de la vie quotidienne, comme le bain, et les activités instrumentales, comme l'utilisation du téléphone, ainsi que les comportements de passage à l'acte, de repli sur soi et d'errance.

Le caractère predictif du diagnostic de démence, des fonctions cognitives et des caractéristiques de comportement peut dépendre du degré de soutien social et de l'assistance que reçoit le sujet. Les ressources sociales comprennent à la fois l'assistance bénévole (assistance reçue de parents, d'amis et de voisins) et l'assistance rémunérée (aide domestique et professionnelle). Nous prévoyons que la relation entre le soutien social et l'occurrence des dommages ne sera pas simple parce que les gens qui reçoivent un plus grand soutien peuvent le recevoir parce qu'ils sont perçus comme plus à risque; en conséquence, le risque de dommages peut être réduit chez ces personnes en raison du soutien qu'elles reçoivent. Il apparait donc clairement que la relation entre les troubles cognitifs, les caractéristiques comportementales, le diagnostic de démence, le soutien du milieu et la survenue de dommages est complexe. Nous devons toutefois démêler cette complexité pour être en mesure de prendre des décisions fondées sur les faits en matière de soins. ${ }^{2}$

\section{Conclusion}

En dehors des études sur le taux d'accident des conducteurs atteints de troubles cognitifs, il n'existe aucune étude portant de façon spécifique sur les problèmes que les personnes âgées atteintes de troubles cognitifs éprouvent en raison de ces troubles. Chaque jour, des médecins, des psychologues, des travailleurs sociaux, des ergothérapeutes et des proches doivent prendre des décisions quant au maintien à domicile de personnes atteintes de troubles cognitifs. Pourtant, ces décisions sont prises sans qu'aucune justification fondée sur des faits ne puisse permettre de prédire la sécurité ou le risque de dommages. Nous ne savons pas si un diagnostic de démence permet de prédire des dommages importants. Nous ne savons pas non plus quel stade de la démence ou du profil d'aptitude cognitive est le plus utile pour la prédiction. La connaissance des comportements présentés par le sujet (errances, hallucinations, etc.) peut aussi constituer un élément critique de la prédiction. De plus, les réseaux de soutien officiels et officieux du sujet devraient atténuer l'influence des caractéristiques diagnostiques, 
comportementales et cognitives sur la survenue de dommages. Il est nécessaire de poursuivre les recherches sur ce point dans plusieurs centres car l'évolution économique et démographique du Canada nous place face au défi d'assurer les soins de la plus haute qualité à nos aînés, tout en respectant leur besoin d'indépendance.

\section{Notes}

1 En Ontario, la personne nommée pour administrer les affaires d'une personne jugée inapte est appelée un «substitute decision-maker". Dans d'autres territoires canadiens, cette fonction peut être assumée par un tuteur, un administrateur ou un curateur de biens.

2 Au Sunnybrook Health Science Centre, nous étudions présentement ce problème dans le cadre d'une étude prospective longitudinale portant sur plus de 200 personnes àgées atteintes de troubles cognitifs et vivant seules. Nous procédons à une évaluation initiale portant sur le diagnostic de démence et sur les caractéristiques cognitives, sociales et comportementales. À la suite de cette évaluation initiale, nous suivons les sujets sur une période de dix-huit mois et enregistrons toute occurrence de dommages importants durant cette période. Nous désirons établir s'il est possible de prédire lesquelles de ces personnes subiront des dommages importants. S'il est possible de développer une prédiction fiable, notre objectif est de développer un outil qui pourra être utilisé par les professionnels de la santé et les organismes communautaires pour diriger leurs ressources limitées vers les personnes qui en ont le plus besoin. L'équipe du centre Sunnybrook comprend les Drs M.C. Tierney, Jocelyn Charles, Susan Jaglal, John P. Szalai, Rory H. Fisher et W. Gary Snow. Ce projet de recherche est financé par la Fondation ontarienne de la santé mentale, Alzheimer Association (U.S.), la Fondation Carolyn Sifton et la Fondation Alva.

\section{Références}

Jacobs, D.M., Sano, M., Dooneief, G., Marder, K., Bell, K., \& Stern, Y. (1995). Neuropsychological detection and characterization of preclinical Alzheimer's disease. Neurology, 45, 957-962.

McFall, S., \& Miller, B.H. (1992). Caregiver burden and nursing home admission of frail elderly persons. Journal of Gerontology, 47, 73-79.

Newman, S.J., Struyk, R., Wright, P., \& Rice, M. (1990). Overwhelming odds: Caregiving and the risk of institutionalization. Journal of Gerontology, 45, 173-183.

Ostbye, T., \& Crosse, E. (1994). Net economic costs of dementia in Canada. Canadian Medical Association, 151, 1457-1464.

Rutman, D., \& Silberfeld, M. (1992). A preliminary report on the discrepency between clinical and test evaluations of competence. Canadian Journal of Psychiatry, 37, 634-639.

Tierney, M.C., Szalai, J.P., Snow, W.G., Fisher, R.H., Nores, A., Nadon, G., Dunn, E., \& St.George-Hyslop, P.H. (1996). Prediction of probable Alzheimer's disease in memory-impaired patients: A prospective longitudinal study. Neurology, 46, 661-665.

Tierney, M.C., Szalai, J.P., Snow, W.G., \& Fisher, R.H. (1996). The prediction of Alzheimer's disease: The role of patient and informant perceptions of cognitive deficits. Arch Neurol, 53, 423-427.

Trobe, J.D., Waller, P.F., Cook-Flannagan, C.A., Teshima, S.M., \& Bieliauskas, L.A. (1996). Crashes and violations among drivers with Alzheimer's disease. Arch Neurol, 53, 411-416. 
Webber, P.A., Fox, P., \& Burnette, D. (1994). Living alone with Alzheimer's disease:

Effects on health and social service utilization patterns. The Gerontologist, 34, 88-14.

Mary C. Tierney 